\title{
Longitudinal Development of Human Brain Wiring Continues from Childhood into Adulthood
}

\author{
Catherine Lebel and Christian Beaulieu \\ Department of Biomedical Engineering, University of Alberta, Edmonton, Alberta T6G 2V2, Canada
}

Healthy human brain development is a complex process that continues during childhood and adolescence, as demonstrated by many cross-sectional and several longitudinal studies. However, whether these changes end in adolescence is not clear. We examined longitudinal white matter maturation using diffusion tensor tractography in 103 healthy subjects aged 5-32 years; each volunteer was scanned at least twice, with 221 total scans. Fractional anisotropy (FA) and mean diffusivity (MD), parameters indicative of factors including myelination and axon density, were assessed in 10 major white matter tracts. All tracts showed significant nonlinear development trajectories for FA and MD. Significant within-subject changes occurred in the vast majority of children and early adolescents, and these changes were mostly complete by late adolescence for projection and commissural tracts. However, association tracts demonstrated postadolescent within-subject maturation of both FA and MD. Diffusion parameter changes were due primarily to decreasing perpendicular diffusivity, although increasing parallel diffusivity contributed to the prolonged increases of FA in association tracts. Volume increased significantly with age for most tracts, and longitudinal measures also demonstrated postadolescent volume increases in several association tracts. As volume increases were not directly associated with either elevated FA or reduced MD between scans, the observed diffusion parameter changes likely reflect microstructural maturation of brain white matter tracts rather than just gross anatomy.

\section{Introduction}

Childhood and adolescence are periods of significant change, with behavioral, emotional, hormonal, and cognitive processes undergoing maturation. Development does not end there, as young adulthood also provides new challenges and experiences that may continue to impact brain development. Function is inherently linked with brain structure, so a detailed knowledge of healthy brain development is crucial for better understanding cognitive and behavioral changes that occur as one ages. Four landmark studies approximately a decade ago (two cross-sectional, two longitudinal) demonstrated significant brain maturation during adolescence and early adulthood using sophisticated image processing of conventional MRI scans (Giedd et al., 1999; Paus et al., 1999; Sowell et al., 1999; Thompson et al., 2000). Since then, additional longitudinal studies using conventional MRI have added substantially to our understanding of healthy brain development within individual subjects (Sowell et al., 2004; Lenroot et al., 2007).

Received Oct. 8, 2010; revised May 29, 2011; accepted June 8, 2011.

Author contributions: C.L. and C.B. designed research; C.L. and C.B. performed research; C.L. and C.B. analyzed data; C.L. and C.B. wrote the paper.

This work was supported by operating grants from the Canadian Institute for Health Research and the Networks of Centres of Excellence-Canadian Language and Literacy Research Network. Salary support was provided by Alberta Innovates-Health Solutions (C.B., C.L.) and Natural Sciences and Engineering Research Council (C.L.). The Canada Foundation for Innovation, Alberta Science and Research Authority, Alberta Heritage Foundation for Medical Research, and the University Hospital Foundation provided infrastructure support. We thank Lindsay Walker, who assisted with initial scanning of some subjects, Saul Caverhill-Godkewitsch and Aaron Deblois for providing volume information, and Dr. Carol Boliek for providing reliability data for four subjects.

Correspondence should be addressed to Dr. Christian Beaulieu, Department of Biomedical Engineering, Faculty of Medicine and Dentistry, Room 1098, Research Transition Facility, University of Alberta, Edmonton, AB T6G 2V2, Canada. E-mail: christian.beaulieu@ualberta.ca.

DOI:10.1523/JNEUROSCI.5302-10.2011

Copyright $\odot 2011$ the authors $\quad 0270-6474 / 11 / 3110937-11 \$ 15.00 / 0$
Sensitive imaging methods such as diffusion tensor imaging (DTI) demonstrate much more widespread changes than conventional MRI in cross-sectional populations. DTI has shown increasing fractional anisotropy (FA), a parameter linked to axon packing and myelination (Beaulieu, 2002), and decreasing mean diffusivity (MD), a parameter reflecting water content and density, throughout brain white matter during childhood, adolescence, and into young adulthood (Mukherjee et al., 2001; Schmithorst et al., 2002; Barnea-Goraly et al., 2005; Ashtari et al., 2007; Bonekamp et al., 2007; Eluvathingal et al., 2007; Lebel et al., 2008a). In general, maturation of commissural and projection fibers occurs earliest, while association fibers continue maturating at later ages, and frontal-temporal connections demonstrate the most prolonged development.

Although informative, cross-sectional studies are limited because they cannot provide information about change within individuals. Two small-sample longitudinal DTI studies in adolescence showed diffusion changes in white matter over relatively short intervals of 1.5-2.5 years (Bava et al., 2010; Giorgio et al., 2010). However, evidence for early childhood and postadolescent longitudinal changes in key brain connections is still lacking. Furthermore, tractography-derived volume is an important parameter that shows age-related changes from adolescence to the elderly (Sala et al., 2010) yet has not been assessed longitudinally. It remains unclear how tract volume changes with age, and longitudinal studies such as this are well poised to examine the relationship between volume and diffusion parameter changes. Understanding healthy development from childhood to young adulthood is critical given that many psychiatric disorders emerge during late adolescence and young adulthood, presumably due to abnormal brain development (Paus et al., 2008). 


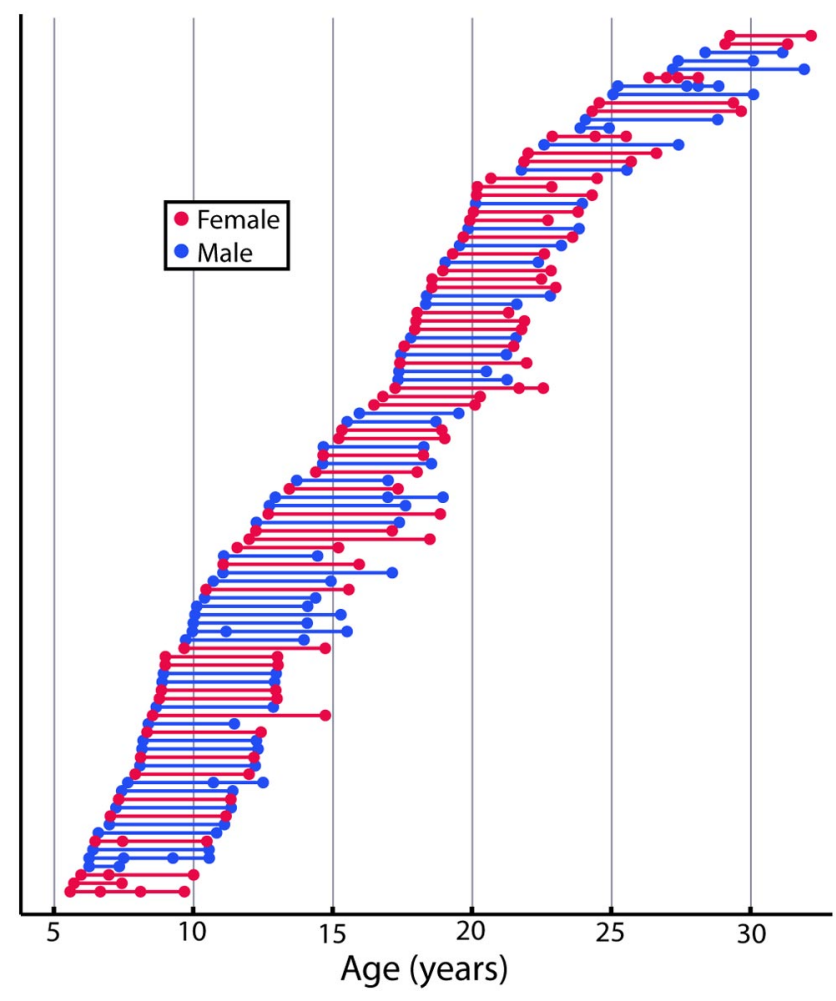

Figure 1. Age at scans for all subjects. Each of the 221 scans obtained is represented by a circle; each of the 103 subjects is shown in a different row, with their scans connected by a straight line. Females (red) and males (blue) are marked separately. Note the relatively even spacing of subjects across the age range, with most subjects receiving two scans $\sim 4$ years apart. Several subjects received three or four scans in total.

Frontal lobe white matter connections are commonly implicated as underdeveloped in psychiatric disorders including schizophrenia (Kubicki et al., 2007), mood disorders (Heng et al., 2010), and anxiety (Kim and Whalen, 2009).

For the first time, longitudinal data are presented for $10 \mathrm{com}$ missural, projection, and association white matter tracts using DTI tractography in a large group of 103 healthy subjects with 221 scans from childhood to young adulthood (5-32 years). This analysis of within-subject diffusion parameter changes over 1-6 year intervals will provide evidence for continued white matter "wiring" development after adolescence, particularly for frontal lobe connections.

\section{Materials and Methods}

Subjects. Subjects for this study were 103 healthy volunteers with no self-reported (or parent-reported) history of neurological or psychiatric disease or brain injury. Subjects were initially aged 5.6-29.3 years (mean age $\pm \mathrm{SD}, 14.8 \pm 6.4$ years; 52 females $/ 51$ males; 97 right-handed $/ 6$ left-handed). A total of 221 scans were obtained on these subjects: 92 volunteers were scanned twice, 7 subjects received three scans, and 4 subjects were scanned four times (Fig. 1). The mean age gap between first and second scans was 4.0 years (range of 1.0-6.5 years); average gaps between first and third scans and first and fourth scans were 4.6 (2.6-6.0 years) and 3.4 years (1.8-4.3 years), respectively. Many of these subjects (94) were included in a previous cross-sectional study of brain development from age 5 to 30 years (Lebel et al., 2008a) and were subsequently scanned several years later. All subjects gave informed consent; child assent and parent/guardian consent was obtained for volunteers under 18 years.

Image acquisition. All data were acquired on the same 1.5 T Siemens Sonata MRI scanner with identical methods. DTI acquisition used a dual spin-echo, single-shot echo-planar imaging sequence with the following parameters: 40 3-mm-thick slices with no interslice gap; TR, $6400 \mathrm{~ms}$;
TE, $88 \mathrm{~ms}$; six noncollinear diffusion sensitizing gradient directions with $b=1000 \mathrm{~s} / \mathrm{mm}^{2}$; eight averages; field of view, $220 \times 220 \mathrm{~mm}^{2}$; matrix of $128 \times 128$ with $75 \%$ phase partial Fourier, zero-filled to $256 \times 256$. Total DTI acquisition time was 6:06 min. To enable measurements of global volume changes, high-resolution $\left(1 \times 1 \times 1 \mathrm{~mm}^{3}\right)$ T1-weighted images were acquired using MPRAGE with TE of $4.38 \mathrm{~ms}$, TR of $1870 \mathrm{~ms}$, TI of $1100 \mathrm{~ms}$; scan time was 4:29 min. T1-weighted images were successfully acquired for 215 of the 221 scans; there were 99 subjects with MPRAGE images at multiple time points.

Tractography. A previously described semiautomated tractography method (Lebel et al., 2008b) was used to delineate 10 major white matter tracts in each individual, according to a priori information on tract location (Wakana et al., 2004). The tracts delineated were as follows: genu, body, and splenium of the corpus callosum, corticospinal tracts, superior and inferior longitudinal fasciculi, superior and inferior fronto-occipital fasciculi, uncinate fasciculus, and cingulum. Fiber tracking was performed using code modified from ExploreDTI (A. Leemans, Utrecht, Netherlands), using a deterministic streamline method. FA thresholds were set to 0.25 to initiate and continue tracking, while the angle threshold was set to $60^{\circ}$ for the uncinate fasciculus and superior longitudinal fasciculus, and $30^{\circ}$ for all other tracts. An FA threshold of 0.25 was chosen to avoid voxels that are not part of the white matter tract (cortex has FA $\sim 0.2$ ), minimize the inclusion of voxels with a higher degree of partial volume contamination, and limit the presence of spurious tracts. Left and right hemisphere fibers were measured separately where appropriate.

Diffusion measurements. FA and MD were measured for each individual scan for each tract by averaging all voxels along the tract; each voxel was counted only once. To investigate the causes of any changes of FA across the age span, the parallel $\left(\lambda_{/ /}=\lambda_{1}\right)$, and perpendicular $\left[\lambda_{\perp}=\left(\lambda_{2}+\lambda_{3}\right) / 2\right]$ diffusivities (i.e., eigenvalues) were also measured for each tract.

Interscan reliability. To test the interscan reliability of FA, MD, parallel and perpendicular diffusivity measurements, seven individuals aged 7, 9, $10,10,17,21$, and 23 years were scanned three to four times each within 6 months. All diffusion parameters values were calculated for each tract according to the semiautomated methods described above. The average SD (average of the SDs measured from each subject) for each parameter for each tract provided estimates for reliability of the diffusion measurements. All of the mean SDs are shown in Table 1. For 8 of 10 tracts, FA SD was $\sim 0.01$ (range, $0.007-0.013$ ); the corticospinal tracts separated into left and right and cingulum had SDs of 0.019 and 0.016 , respectively. Most MD SDs were $0.010-0.012 \times 10^{-3} \mathrm{~mm}^{2} / \mathrm{s}$, although the SDs were higher $\left(0.017-0.025 \times 10^{-3} \mathrm{~mm}^{2} / \mathrm{s}\right)$ for all three sections of the corpus callosum and the corticospinal tracts.

The cingulum had the highest coefficient of variation for FA, at 0.032 , while coefficients of variation for FA in the other tracts ranged from 0.015 in the inferior longitudinal fasciculus to 0.025 in the corticospinal tract. Variation was similar for MD: the corticospinal tract had the highest variation at 0.030 , while the corpus callosum variation was $0.022-0.029$, and the association tracts ranged were all 0.014-0.015.

Statistical analysis. Age-related changes of FA, MD, and parallel and perpendicular diffusivity were analyzed using linear mixed models to account for the repeated measurements on each individual. Initially, males and females were fit separately with quadratic curves. However, as the age and age $^{2}$ terms (representing curve shape) were not significantly different between genders for any tract for either FA or MD, genders were combined for further analysis, but a sex term was included to account for any absolute differences based on gender. The following equation was fit to the data: FA $($ or $\mathrm{MD})=A \cdot$ age $^{2}+B \cdot$ age $+C+D \cdot$ sex. If the age ${ }^{2}$ term was not significant, a linear fit was calculated. Initially, separate fits were calculated for the left and right hemisphere tracts, where appropriate. If the age and age ${ }^{2}$ fitting parameters were not significantly different between hemispheres (using $F$ tests), average values were computed and one fit was done for both left and right sides combined. To increase power and reduce the total number of variables, genders and hemispheres were combined for analysis of perpendicular and parallel diffusivity.

To evaluate longitudinal changes within individuals, subjects were divided into six groups according to age: $5-11$ years ( 15 subjects initially 
Table 1. Tractography-derived SDs over multiple scans

\begin{tabular}{|c|c|c|c|c|c|}
\hline Tract & FA & $\mathrm{MD}\left(\times 10^{-3} \mathrm{~mm}^{2} / \mathrm{s}\right)$ & $\lambda_{/ /}\left(\times 10^{-3} \mathrm{~mm}^{2} / \mathrm{s}\right)$ & $\lambda_{\perp}\left(\times 10^{-3} \mathrm{~mm}^{2} / \mathrm{s}\right)$ & Volume $\left(\mathrm{cm}^{3}\right)$ \\
\hline Genu of the corpus callosum & 0.012 & 0.017 & 0.023 & 0.016 & 1.15 \\
\hline Body of the corpus callosum & 0.013 & 0.022 & 0.034 & 0.020 & 2.29 \\
\hline Splenium of the corpus callosum & 0.012 & 0.024 & 0.032 & 0.022 & 1.06 \\
\hline Corticospinal tract & 0.019 & 0.025 & 0.030 & 0.025 & 1.23 \\
\hline Cingulum & 0.016 & 0.012 & 0.020 & 0.017 & 0.54 \\
\hline Inferior longitudinal fasciculus & 0.007 & 0.012 & 0.017 & 0.012 & 1.27 \\
\hline Inferior fronto-occipital fasciculus & 0.012 & 0.012 & 0.022 & 0.011 & 2.65 \\
\hline Superior longitudinal fasciculus & 0.009 & 0.010 & 0.012 & 0.012 & 1.25 \\
\hline Superior fronto-occipital fasciculus & 0.009 & 0.011 & 0.013 & 0.012 & 0.72 \\
\hline Uncinate fasciculus & 0.010 & 0.012 & 0.025 & 0.022 & 0.83 \\
\hline
\end{tabular}

Seven subjects were scanned three to four times each within 6 months to determine reliability of the diffusion and tract volume measurements over time. The mean of the SDs over all scans per subject are shown for each parameter [FA, MD, parallel $\left(\lambda_{/ /}\right)$and perpendicular diffusivity $\left(\lambda_{\perp}\right)$, and tract volume], which were then used as a threshold for determining whether the parameters increased or decreased between scans in the study population.
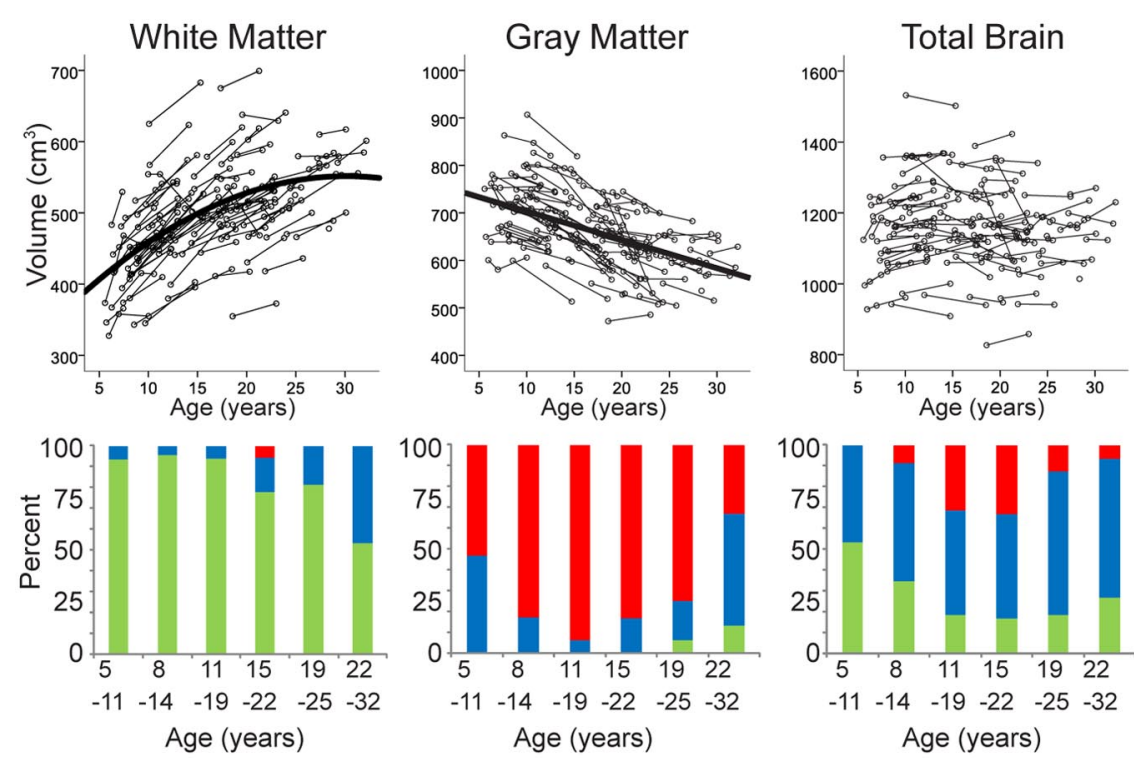

Percent of subjects with

Volume Decrease

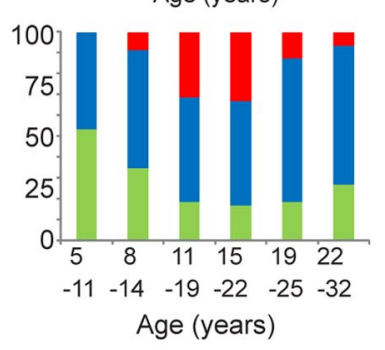

Volume Increase

Figure 2. Longitudinal volume changes on T1-weighted images. Changes of white matter, gray matter, and total brain volume with age for each scan are shown (top row) along with bar graphs reflecting the percentage of subjects with volume increases (green), decreases (red), or no change (blue) within six age categories. White matter volume increased significantly across the age range, including the twenties, while gray matter volume decreased in the majority of children, adolescents, and young adults up to 25 years. These white matter increases and gray matter decreases offset one another such that total brain volume did not change in most persons, although many of the younger subjects had small increases.

aged $5-8$ years and aged $7-11$ years at their final scan), $8-14$ years $(8-10$ to $12-14$ years; $n=23), 11-19$ years $(11-14$ to $15-19$ years; $n=16)$, $15-22$ years ( $15-18$ to $19-22$ years; $n=18), 19-25$ years $(19-21$ to $22-25$ years; $n=16)$, and $22-32$ years $(22-29$ to $24-32$ years; $n=15)$. For subjects with only two scans, the change of FA or MD within each region was measured as the value at the second scan minus the value at the first. For subjects with more than two scans, best fit lines were calculated, and the total diffusion parameter changes were measured as the difference between the best fit line at the final age and the initial age. Within each age group, the number of subjects who had FA or MD increases or decreases greater than the average SD for that tract (as computed by the reliability tests described above and shown in Table 1) were tabulated. Subjects who had FA or MD changes within $1 \mathrm{SD}$ were considered not to change.

Brain volume. To yield global brain metrics, gray matter and white matter volume, as well as total brain volume were measured using FreeSurfer (Athinoula Martinos Center for Biomedical Imaging, Charlestown, MA). Total brain volume did not include CSF. Changes in brain volumes were modeled using the same linear mixed-models methods described above for FA and MD changes. In addition, volume changes were analyzed in individual subjects (by age group), as for FA and MD values. Since reliability measures were not available for overall volumes

(T1-weighted scans were not obtained in the earlier DTI reliability assessments), any changes $>2 \%$ of the total volume were considered "increases" or "decreases"; variations of $<2 \%$ were considered to be no change.

Tract volume. Tract volume for each of the white matter fiber bundles was measured by summing the total number of voxels through which at least one streamline passed. It is important to note that this volume measure does not necessarily correspond to the actual tract volume, but is rather a measure of the number of voxels attributed to a given tract that exceeds the used FA threshold. Tract volume was tested for age-related changes in the same manner as FA and MD, first using a mixed-models analysis to determine the development trajectory, and second using a group analysis to look at within-subject changes based on reliability measures (calculated as the SDs from seven volunteers scanned multiple times) shown in Table 1. To assess the relationship between tract volume and diffusion parameters, volume for each tract was included as a covariate in the mixed-models analysis for FA and MD. As the within-subject changes are more informative, volume changes were also evaluated within subjects who had increasing, decreasing, or stagnant FA and MD values between scans to determine what proportion also had volume increases/decreases.

\section{Results}

Brain volume

Despite a lack of significant overall brain volume changes, white matter volume increased significantly across the age range of 5-32 years while there was a concomitant decrease in gray matter volume (Fig. 2). When individual subjects were examined, almost all of the younger subjects $(>95 \%)$ demonstrated increases of white matter volume from their first to last scan, and the majority of subjects $(50-80 \%)$ in the older age groups (15-32 years, representing adolescence and young adulthood) continued to demonstrate white matter volume increases between scans ( $\sim 4$ years apart on average). The white matter increases were offset by gray matter decreases, leading to no overall significant change in total brain volume with age.

\section{Mixed-models analysis of FA and MD changes}

DTI tractography provides more specific information about individual fibers than global white matter volume. Using a mixedmodels approach, age-related changes in diffusion parameters 
Table 2. Fitting parameters and $p$ values for age-related changes of FA and MD for all 10 white matter fibers

\begin{tabular}{|c|c|c|c|c|c|c|c|c|c|}
\hline & \multicolumn{5}{|c|}{ Fractional anisotropy } & \multicolumn{4}{|c|}{ Mean diffusivity $\left(\mathrm{mm}^{2} / \mathrm{s}\right)$} \\
\hline & Intercept & Age $\left(\times 10^{-2}\right)$ & $\operatorname{Age}^{2}\left(\times 10^{-3}\right)$ & $\operatorname{Sex}^{a}$ & & Intercept $\left(\times 10^{-3}\right)$ & Age $\left(\times 10^{-5}\right)$ & $\operatorname{Age}^{2}\left(\times 10^{-6}\right)$ & $\operatorname{Sex}\left(\times 10^{-5}\right)^{a}$ \\
\hline \multicolumn{10}{|l|}{ Commissural tracts } \\
\hline Genu of corpus callosum & $0.52<0.001$ & $0.51<0.001$ & $-0.13<0.001$ & n.s. & & $0.88<0.001$ & $-0.87<0.001$ & $\begin{array}{l}0.18<0.001 \\
0.16 \quad 0.004\end{array}$ & n.s. \\
\hline Body of corpus callosum & $0.48<0.001$ & $0.60<0.001$ & $-0.14<0.001$ & n.s. & & $0.97<0.001$ & $-0.97<0.001$ & & n.s. \\
\hline Splenium of corpus callosum & $0.53<0.001$ & $0.52 \quad 0.001$ & $-0.11 \quad 0.013$ & 0.013 & 0.001 & $0.93<0.001$ & $-1.36<0.001$ & $0.31<0.001$ & n.s. \\
\hline \multicolumn{10}{|l|}{ Projection tracts } \\
\hline $\begin{array}{l}\text { Corticospinal tracts } \\
\text { Left CST }^{b} \\
\text { Right CST }\end{array}$ & $\begin{array}{l}0.43<0.001 \\
0.46<0.001\end{array}$ & $\begin{array}{l}1.03<0.001 \\
0.60<0.001\end{array}$ & $\begin{array}{l}-0.24<0.001 \\
-0.13<0.001\end{array}$ & $\begin{array}{l}-0.009 \\
-0.011\end{array}$ & $\begin{array}{l}0.004 \\
0.001\end{array}$ & $0.86<0.001$ & $-0.20<0.001$ & n.s. & 1.20 .007 \\
\hline \multicolumn{10}{|l|}{ Association tracts } \\
\hline Cingulum & $0.43<0.001$ & $0.54<0.001$ & $-0.07 \quad 0.038$ & -0.014 & $<0.001$ & $0.88<0.001$ & $-0.94<0.001$ & $0.17<0.001$ & 0.890 .021 \\
\hline Inferior longitudinal fasciculus & $0.41<0.001$ & $0.47<0.001$ & $-0.01 \quad 0.001$ & n.s. & & $0.93<0.001$ & $-0.79<0.001$ & $0.16<0.001$ & n.s. \\
\hline Inferior fronto-occipital fasciculus & $0.45<0.001$ & $0.68<0.001$ & $-0.13<0.001$ & n.s. & & $0.88<0.001$ & $-0.68<0.001$ & $0.15<0.001$ & n.s. \\
\hline Superior longitudinal fasciculus & $0.42<0.001$ & $0.67<0.001$ & $-0.14<0.001$ & -0.005 & 0.027 & $0.85<0.001$ & $-0.89<0.001$ & $0.17<0.001$ & n.s. \\
\hline Superior fronto-occipital fasciculus & $0.41<0.001$ & $0.53<0.001$ & $-0.13<0.001$ & n.s. & & $0.82<0.001$ & $-0.62<0.001$ & $0.11 \quad 0.002$ & 0.810 .017 \\
\hline Uncinate fasciculus & $0.37<0.001$ & $0.44<0.001$ & $-0.08 \quad 0.011$ & -0.009 & 0.002 & $0.90<0.001$ & $-0.58<0.001$ & $0.10<0.001$ & n.s. \\
\hline
\end{tabular}

CST, Corticospinal tract; $n$.s., not significant.

${ }^{a}$ Positive values for sex term indicate higher $F A$ or MD values in females.

${ }^{b}$ The CST was assessed separately for each hemisphere for FA measures, where there were significant differences in the age and age ${ }^{2}$ parameters; for MD, there were no significant differences, so hemispheres were combined.

were modeled for each tract. All 10 white matter fibers demonstrated significant age-related changes of FA and MD (Table 2; Figs. 3, 4). In all cases except the corticospinal tract (which had linearly decreasing MD), FA and MD showed nonlinear trends, with more rapid changes at early ages (increases for FA, decreases for $\mathrm{MD}$ ), and slower changes or apparent leveling off during young adulthood. The development curves slow or slightly reverse at the upper end of the age range, but this may not represent the changes occurring within individual subjects. Thus, an additional analysis was conducted to examine white matter evolution within each individual.

\section{Commissural and projection fibers}

In agreement with the mixed-models analysis curves, all three sections of the corpus callosum (genu, body, and splenium) as well as the corticospinal tract had large proportions of younger subjects with FA increases (Fig. 3) and MD decreases (Fig. 4). For all of these tracts, the majority of adolescent and young adult subjects showed no changes of diffusion parameters, although $\sim 20-30 \%$ had decreasing FA or increasing MD, parameter changes usually interpreted as detrimental in elderly aging.

In general, the extent of the commissural fiber tracking was consistent across the sample. The corticospinal tracts tend to be more difficult to track and showed variation among individuals in terms of length. However, the extent and shape of the corticospinal tracts (and other tracts) still tended to be consistent between scans within any particular individual, as can be seen in Figure 5.

\section{Association fibers}

While most adolescent and young adult subjects had few changes in commissural and projection fibers, many had substantial changes in association fibers over 1-6 year intervals. The inferior and superior longitudinal and inferior fronto-occipital fasciculi had the largest proportions of young adults with increasing FA; $\sim 40 \%$ of subjects in the oldest two groups (19-25 and 22-32 years) demonstrated FA increases, while only $10 \%$ had FA decreases between scans. The superior fronto-occipital fasciculus and the cingulum had $\sim 30 \%$ of young adults with FA increases, and an equal proportion with decreasing FA. And finally, the uncinate fasciculus, a frontal-temporal connection, had the few- est adult subjects with FA increases of the association tracts, at $<20 \%$. MD changes show a similar pattern, with the superior and inferior longitudinal fasciculi demonstrating the most prolonged changes with $40-50 \%$ of adult subjects having decreased MD. The superior and inferior fronto-occipital fasciculi also had many young adults with decreasing $\mathrm{MD}(\sim 30 \%)$, although the cingulum and uncinate had more even proportions of subjects with either increasing or decreasing MD.

Association fibers were tracked reliably and consistently across the sample. Two of the more variable tracts (the superior and inferior longitudinal fasciculus) are shown, along with corticospinal tracts, in Figure 5 for four individuals at two time points. The extent of the superior longitudinal fasciculus is variable among individuals, particularly the frontal-temporal section, but appears relatively similar across scans within the same individuals.

\section{Parallel and perpendicular diffusivities}

Four examples of diffusivity changes are shown in Figure 6. For commissural fibers (body of the corpus callosum changes shown in Fig. 6; genu and splenium not shown), the early increases of FA were driven primarily by decreasing $\lambda_{\perp} ; \lambda_{/ /}$also decreased in many subjects. Less than one-third of adolescent and young adult subjects had FA increases in the corpus callosum, and similar proportions had decreases of either parallel or perpendicular diffusivity. In the corticospinal tract (Fig. 6), the FA changes were influenced by both $\lambda_{\perp}$ and $\lambda_{/ /}$, as $\sim 40 \%$ of young subjects have increasing $\lambda_{/ /}$and $25 \%$ have decreasing $\lambda_{\perp}$ between scans; in the adult subjects, approximately equal numbers of subjects have increasing and decreasing diffusivities, with the majority experiencing no change for both $\lambda_{\perp}$ and $\lambda_{/ /}$.

The association tracts showed very similar patterns for perpendicular diffusivity to those of the corpus callosum; the majority $(50-80 \%)$ of the young subjects had decreases of $\lambda_{\perp}$ between scans, and as age went up, fewer subjects experienced decreases of $\lambda_{\perp}$, with the oldest subjects being split fairly evenly between those with decreases, increases, and no change of $\lambda_{\perp}$. Parallel diffusivity had more variation among association tracts. The cingulum (shown in Fig. 6) and the superior fronto-occipital fasciculus showed little change overall of $\lambda_{/ /}$, with some subjects increasing, some decreasing, and many staying the same across the age range. 

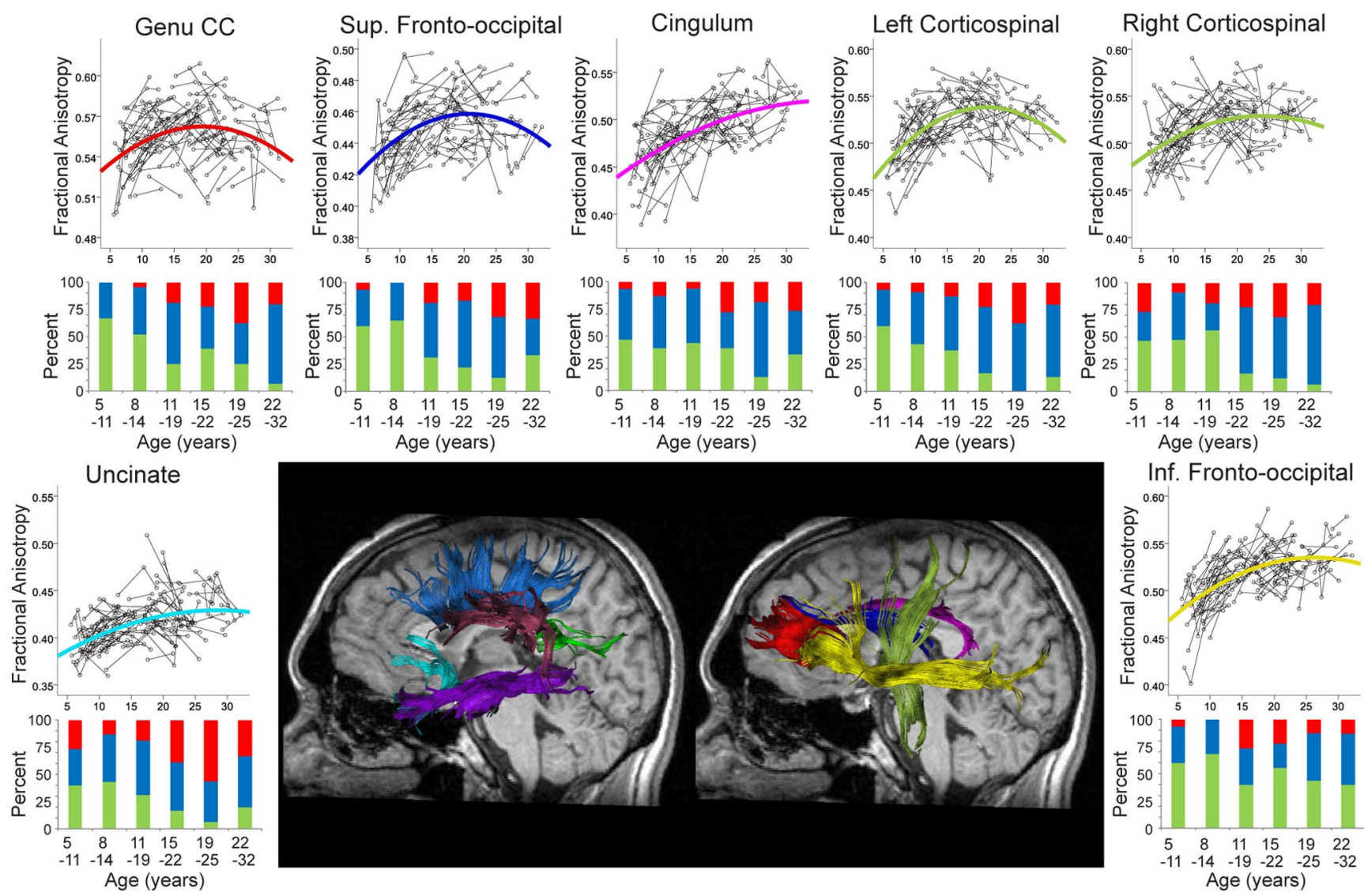

Body CC

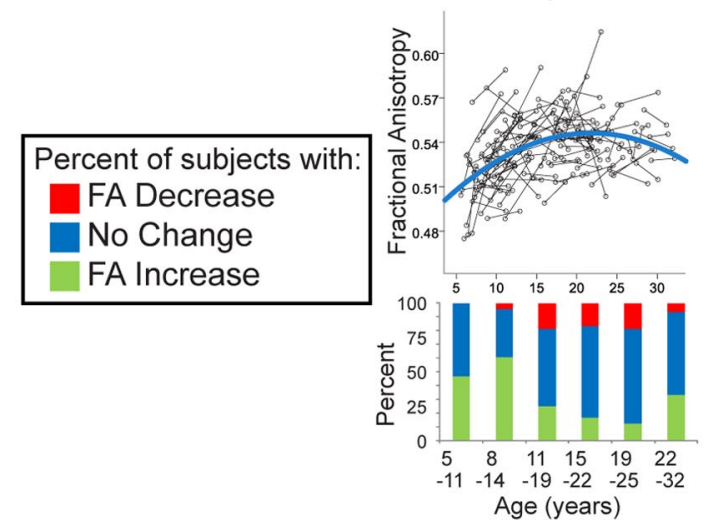

Inf. Longitudinal

Splenium CC

Sup. Longitudinal
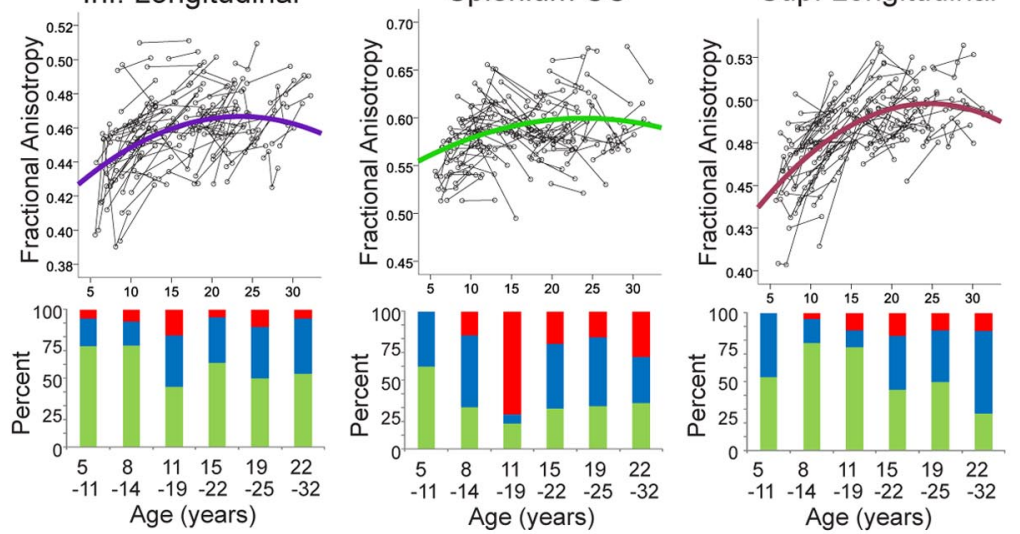

Figure 3. Longitudinal age-related changes of fractional anisotropy. Individual FA data from 221 scans and bar graphs depicting the percentage of 103 subjects whose FA increased (green), decreased (red), or did not change (blue) in six age groupings are shown for all 10 white matter fibers. All fibers showed significant nonlinear increases of $F A$ with age. In callosal and projection fibers, children and young adolescents had FA increases, while most young adults showed no change of FA (although $30 \%$ had increased FA of the body and splenium in $22-32$ year group). The majority of young subjects also had FA increases in association fibers, yet importantly these fibers, such as the superior and inferior fronto-occipital, inferior longitudinal, and cingulum, continue to have $30-50 \%$ of subjects with FA increases between scans even in the 19-25 and 22-32 year age groups. Tracts displayed are from a 22-year-old male.

Interesting patterns were seen, however, in the inferior frontooccipital and longitudinal fasciculi, the superior longitudinal fasciculus and the uncinate fasciculus. In these tracts, the two youngest groups of subjects had the largest proportions $(\sim 40 \%)$ with reductions of parallel diffusivity, but in the next three older age groups, which span adolescence and early adulthood, 40$70 \%$ of the subjects experienced an increase of $\lambda_{/ /}$, while very few had decreases. The oldest age group (22-32 years) was more variable with approximately equal proportions with increasing or decreasing $\lambda_{/ /}$, although approximately one-third to one-half of the subjects still had decreasing $\lambda_{\perp}$.

\section{Sex differences}

Three tracts had sex differences for FA only, one tract had sex differences of MD values only, and two had sex differences of both parameters (Table 2). Females had significantly higher FA values in the splenium of the corpus callosum (by 0.013 units or $2.2 \%)$ while males had significantly higher FA in the cingulum $(0.014 ; 2.9 \%)$, corticospinal tracts (both left and right sides at 0.009 and 0.011 , or 1.7 and $2.1 \%$, respectively), superior longitudinal fasciculus $(0.005 ; 1 \%)$, and uncinate fasciculus $(0.009$; $2.2 \%)$. In terms of $\mathrm{MD}$, females had higher values in the cingulum $\left(0.0089 \times 10^{-3} \mathrm{~mm}^{2} / \mathrm{s} ; 1.1 \%\right)$, corticospinal tracts 

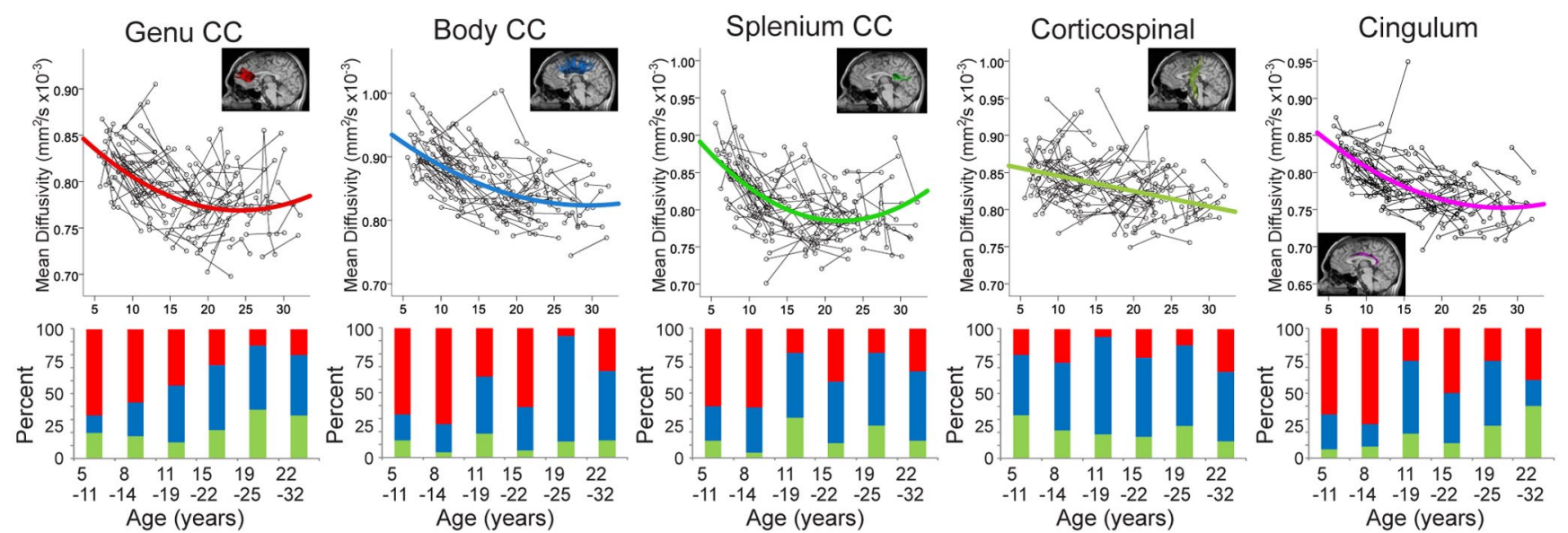

Sup. Longitudinal

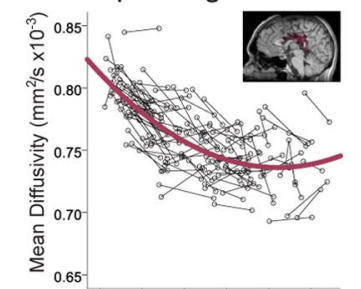

Sup. Fronto-occipital
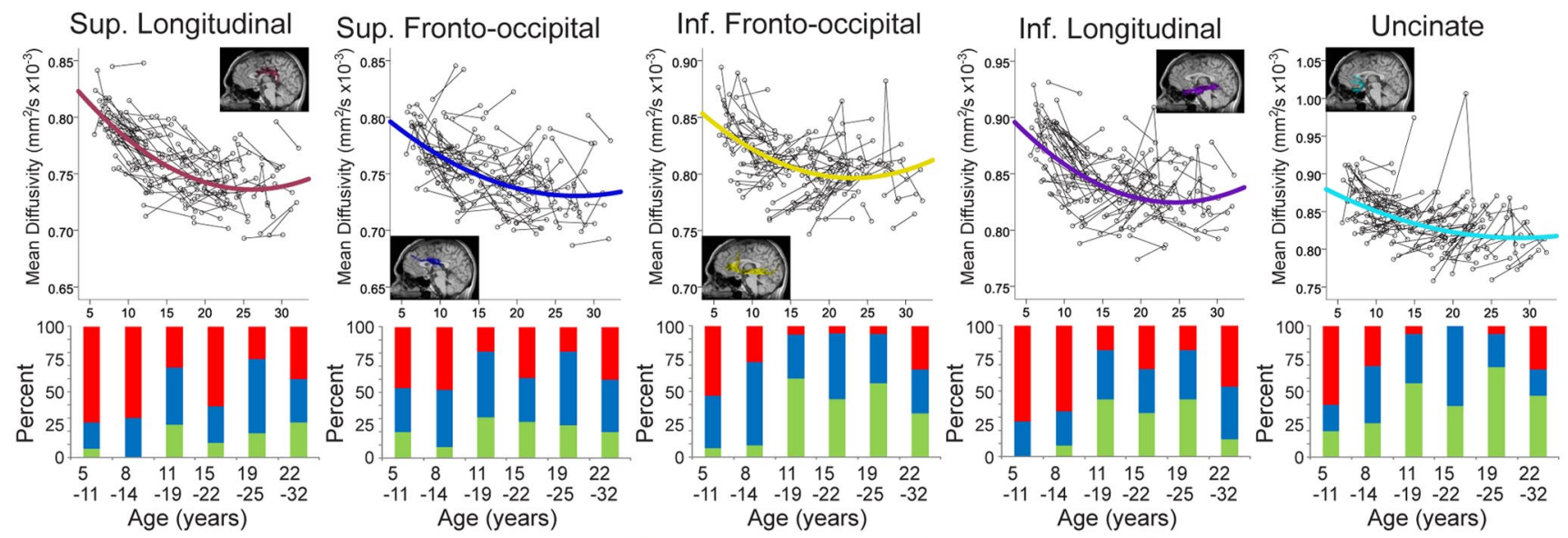

Percent of subjects with:

MD Decrease

No Change

MD Increase

Figure 4. Longitudinal age-related changes of mean diffusivity. All 10 tracts had significant age-related decreases of MD. Commissural tracts demonstrated expected patterns, where a large proportion of subjects had decreasing MD in childhood and there were fewer subjects with decreases at older ages. Most association tracts, however, demonstrated more prolonged MD decreases, with $30-40 \%$ of older subjects having decreases of MD between scans. Notably, the inferior fronto-occipital, inferior longitudinal, and uncinate fasciculi show $40-60 \%$ of subjects with increasing MD between scans as early as the 11-19 year group. Other frontal lobe connecting white matter fibers, such as the genu of the corpus callosum and the cingulum, demonstrate $30-40 \%$ of subjects with increasing MD but only in the oldest age grouping of 22-32 years suggesting age-related decline is beginning in some subjects.

$\left(0.012 \times 10^{-3} \mathrm{~mm}^{2} / \mathrm{s} ; 1.4 \%\right)$, and superior fronto-occipital fasciculus $\left(0.0081 \times 10^{-3} \mathrm{~mm}^{2} / \mathrm{s} ; 1.1 \%\right)$. When examining the within-subject changes, males and females had remarkably similar profiles across the age groups for both diffusion and global volume changes (data not shown).

\section{Tract volume}

Tract volume changed significantly with age for 9 of 10 tracts, with the exception being the superior fronto-occipital fasciculus (Fig. 7). For the genu of the corpus callosum, cingulum, and the inferior longitudinal fasciculus, age-volume fits were quadratic, whereas positive linear fits were best for the body of the corpus callosum, corticospinal tract, superior longitudinal, uncinate, and inferior fronto-occipital fasciculi. Most tracts had volume increases of $63-183 \%$ across the full age span of 5-32 years; the genu had increases of $15 \%$ followed by decreases of $12 \%$. The splenium was the only tract with a linear volume decrease across the age range (total decrease, $41 \%$ ), although a greater proportion of younger subjects had decreases than older subjects. Three tracts in particular had many older subjects with volume increases; for the body of the corpus callosum, the cingulum, and the inferior longitudinal fasciculus, $40-53 \%$ of subjects in the oldest group showed volume increases.

\section{Influence of tract volume on FA and MD changes}

When tract volume was included as a covariate in the mixedmodels analysis of FA and MD, age-related changes were somewhat mitigated, but age remained a significant predictor of FA and MD for all tracts. For within-subject volume changes, Figure 8 shows the proportions of subjects with increasing, decreasing, or stagnant FA and MD values who also had volume increases or decreases. Across the 10 tracts, volume increased in $12-59 \%$ (mean \pm SD, $27 \pm 15 \%$ ) of subjects who had elevated FA between scans; however, $4-49 \%$ (40 $\pm 14 \%)$ of subjects with increasing FA had volume decreases. Of those with decreasing FA values between scans, $8-82 \%(40 \pm 14 \%)$ had volume increases and $4-49 \%$ (29 $\pm 13 \%)$ had volume decreases. Most tracts appeared to have relatively similar proportions of subjects with increasing or decreasing volume for each FA category, although notable exceptions were the cingulum and the body of the corpus callosum, where a higher proportion of subjects with decreasing FA had increasing volumes. Compared with the elevated FA case, more subjects with decreasing MD had volume increases, specifically, $22-74 \%(44 \pm 16 \%)$, while a smaller proportion at $4-41 \%(15 \pm 11 \%)$ had volume decreases. Of subjects with increasing MD, 8-58\% (27 $\pm 15 \%)$ also had increasing volume and $4-49 \%(29 \pm 13 \%)$ had volume de- 


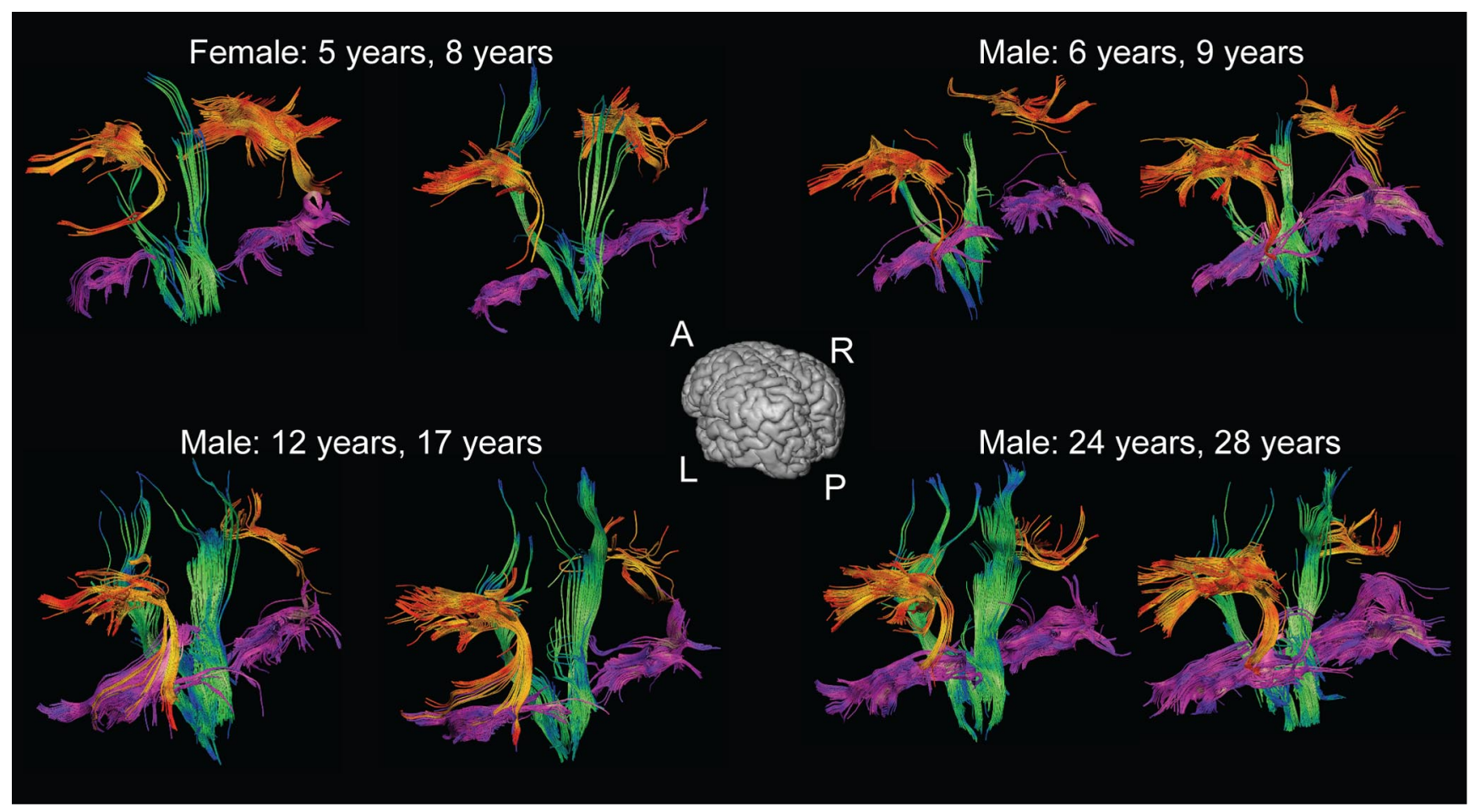

Figure 5. Sample tracts at two time points. Tracts are shown at two time points for several individuals. The superior longitudinal fasciculus (orange), corticospinal tract (green), and inferior longitudinal fasciculus (purple) are given as examples because they can be some of the more difficult and inconsistent fibers to track. Note that, although there are considerable variations in length, size, and shape of the tracts among individuals, the tracts look relatively similar within the same individual.

creases. Overall, most tracts (with the exception of the splenium) had a greater proportion of subjects with increasing volume, as opposed to decreasing volume, associated with elevated FA or reduced MD. However, in both cases, a significant proportion of subjects (27-61\% for elevated FA; $20-57 \%$ for reduced MD) had no change in volume.

\section{Discussion}

In the first longitudinal tractography study of brain development from childhood to adulthood, within-subject maturation is demonstrated during childhood, adolescence, and young adulthood. Development occurs for most children across all 10 major white matter tracts measured, and continues during the twenties in several association tracts, namely the inferior and superior longitudinal and fronto-occipital fasciculi. This extent of maturation was not evident in previous cross-sectional DTI studies in which development of these tracts was considered finished by the twenties (Ben Bashat et al., 2005; Lebel et al., 2008a), although one cross-sectional study noted maturation of the superior longitudinal fasciculus during young adulthood (Giorgio et al., 2008). Frontal connections including these association tracts support complex cognitive processing (Moll et al., 2005; Blakemore and Choudhury, 2006; Jung and Haier, 2007), much of which continues developing during late adolescence and early adulthood. As structural brain changes can be training induced [e.g., juggling in young adults (Scholz et al., 2009)], postadolescent development may be influenced by complex and demanding life experiences such as advanced education, full-time employment, independence, and new social/family relationships.

In agreement with previous longitudinal brain volume studies (Giedd et al., 1999; Sowell et al., 2004; Lenroot et al., 2007), we show white matter increases and gray matter decreases in most subjects across the age range, including many young adults (Fig. 2). Although
T1-weighted MRI volume measures are based on tissue contrast and may have errors introduced by image quality that differ with age, results here are compatible with autopsy studies suggesting continued myelination and decreased synaptic density during adolescence (Yakovlev and Lecours, 1967; Huttenlocher, 1979).

DTI, which provides a more sensitive measure of white matter development than overall volume, demonstrated nonlinear trajectories of FA and MD across the age span (Figs. 3, 4), which are in good agreement with cross-sectional DTI studies showing agerelated changes through childhood and adolescence (Mukherjee et al., 2001; Schmithorst et al., 2002; Barnea-Goraly et al., 2005; Ben Bashat et al., 2005; Ashtari et al., 2007; Bonekamp et al., 2007; Eluvathingal et al., 2007), and implying continued development beyond adolescence (Faria et al., 2010; Westlye et al., 2010). Two previous longitudinal DTI studies on 22/24 subjects spanning ages 16-21/13-19 years also demonstrate brain maturation during adolescence (Bava et al., 2010; Giorgio et al., 2010). Using a larger age range and sample, we show within-subject brain development during young adulthood in association tracts, particularly frontal connections needed for complex cognitive tasks such as inhibition, executive functioning, and attention.

Group analysis of within-subject changes revealed interesting results not evident in the mixed-models trajectories. In all tracts, most children had increasing FA and decreasing MD between scans, demonstrating widespread maturation. In older age groups, most tracts had progressively fewer subjects with FA increases and more with FA decreases (Fig. 3). However, some association tracts demonstrate prolonged postadolescent maturation: $>40 \%$ of subjects in the oldest group (22-32 years) had increasing FA in the inferior longitudinal and fronto-occipital fasciculi, and decreasing MD in the superior and inferior longitudinal, the superior fronto-occipital, and the cingulum. 


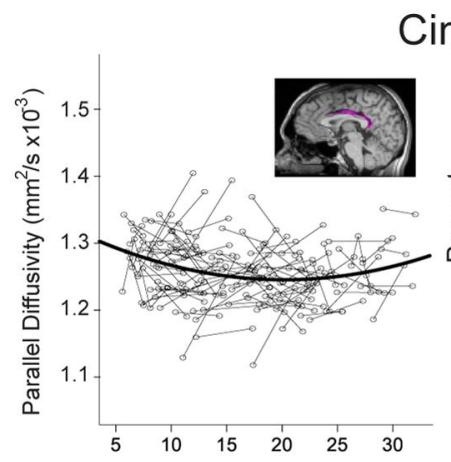

Cingulum

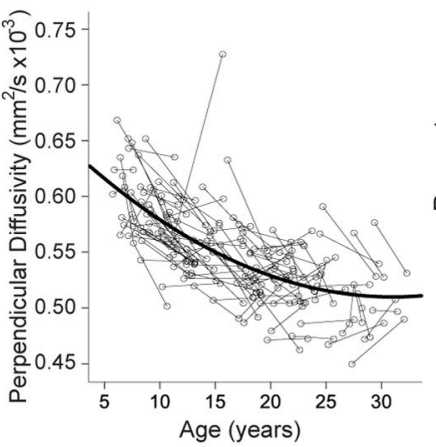

Body of the Corpus Callosum
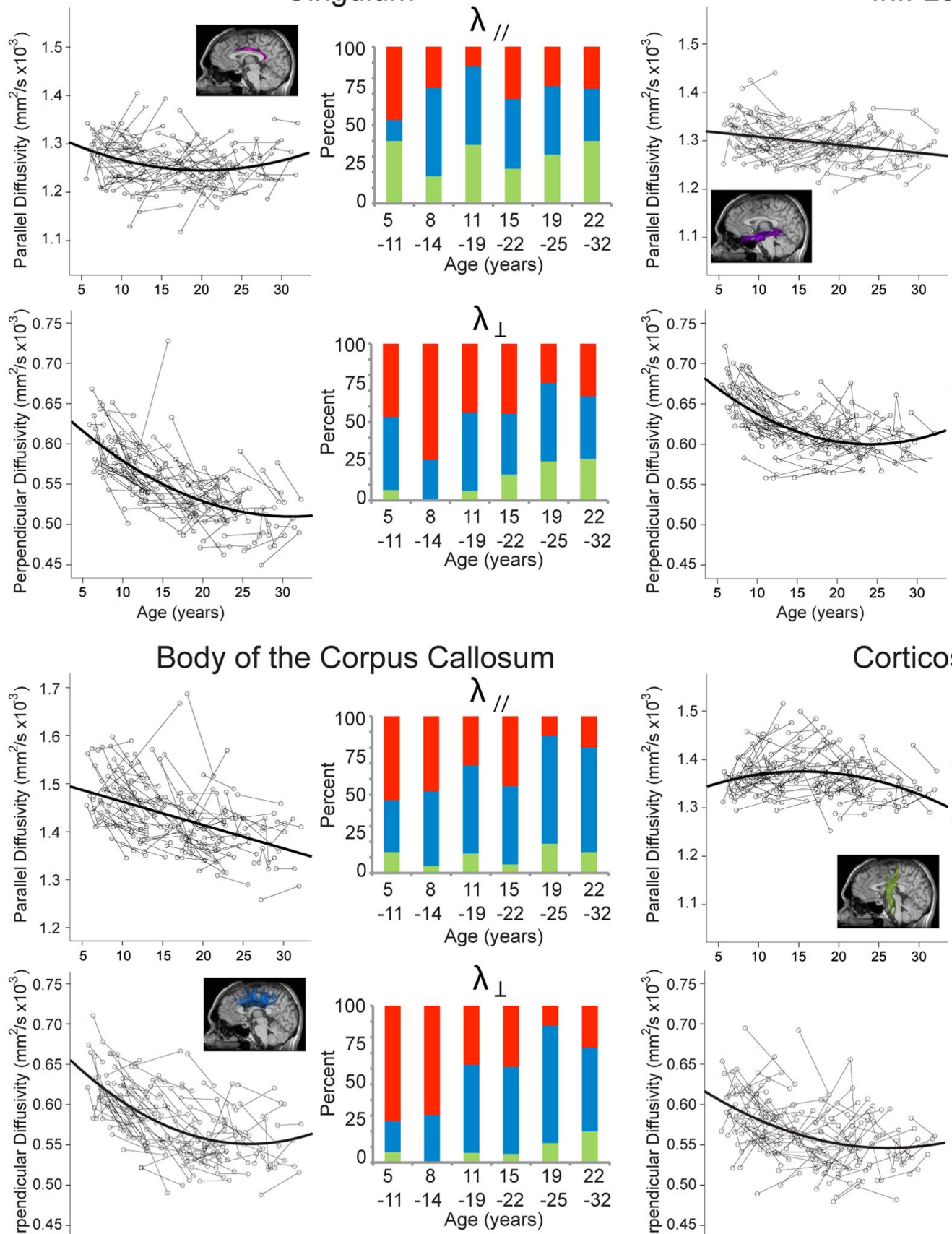

Inf. Longitudinal
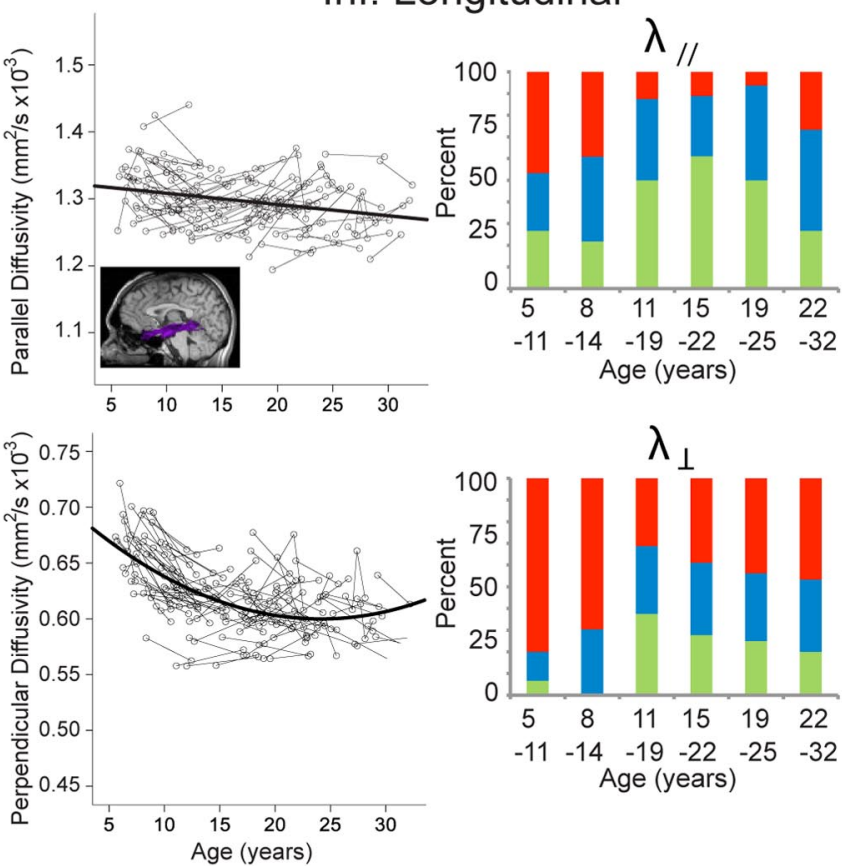

Corticospinal Tract
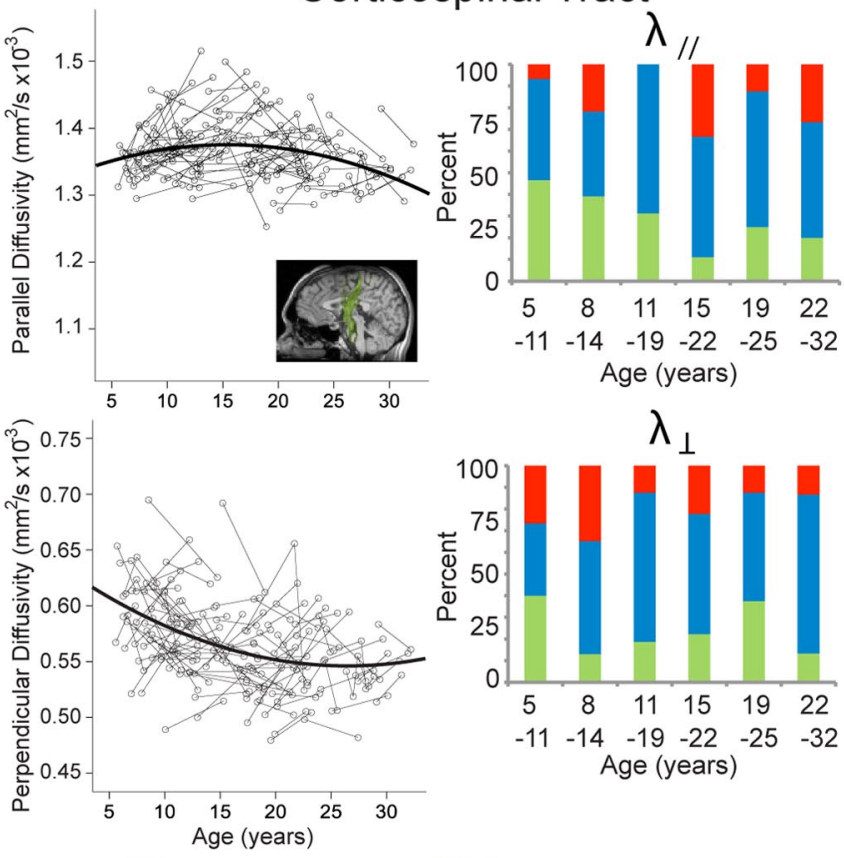

No Change

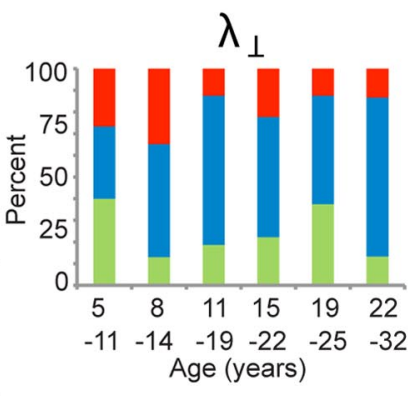

Diffusivity Increase

Figure 6. Longitudinal changes of parallel and perpendicular diffusivity. For all tracts, most young subjects had decreased perpendicular diffusivity between scans and fewer older subjects had decreases. Parallel diffusivity trajectories varied more among tracts. Most association fibers (such as the cingulum shown above) had only small changes of parallel diffusivity, although for tracts with prolonged FA changes (superior and inferior longitudinal, shown above, and inferior fronto-occipital fasciculi), a substantial portion of older subjects demonstrated increased parallel diffusivity between scans. Commissural fibers had substantial decreases of both parallel and perpendicular diffusivity in young subjects, with most subjects having no change at older ages. The corticospinal tract was unique in that its parallel diffusivity trajectory increased at young ages; its perpendicular diffusivity trajectory was similar to that of other tracts.

Changes within individuals were relatively small, averaging 5-7\% for the youngest subjects and $4 \%$ in the oldest age group. Since the biggest surge of brain development occurs before the age of 5 years (Hermoye et al., 2006; Dubois et al., 2008; Faria et al., 2010), these fairly small changes are not surprising.

An important observation in this study is that many, but not all, young adult subjects have increasing FA. For example, 40-
$50 \%$ of subjects from 19 to 32 years have increasing FA in the inferior longitudinal and fronto-occipital fasciculi, demonstrating prolonged development in these regions; however, 5-15\% have decreased FA between scans. This suggests interesting between-subject differences (evident in scatter plots in Figs. 3 and 4) in the progression of brain structure and may be valuable for understanding the relationship between psychiatric disor- 

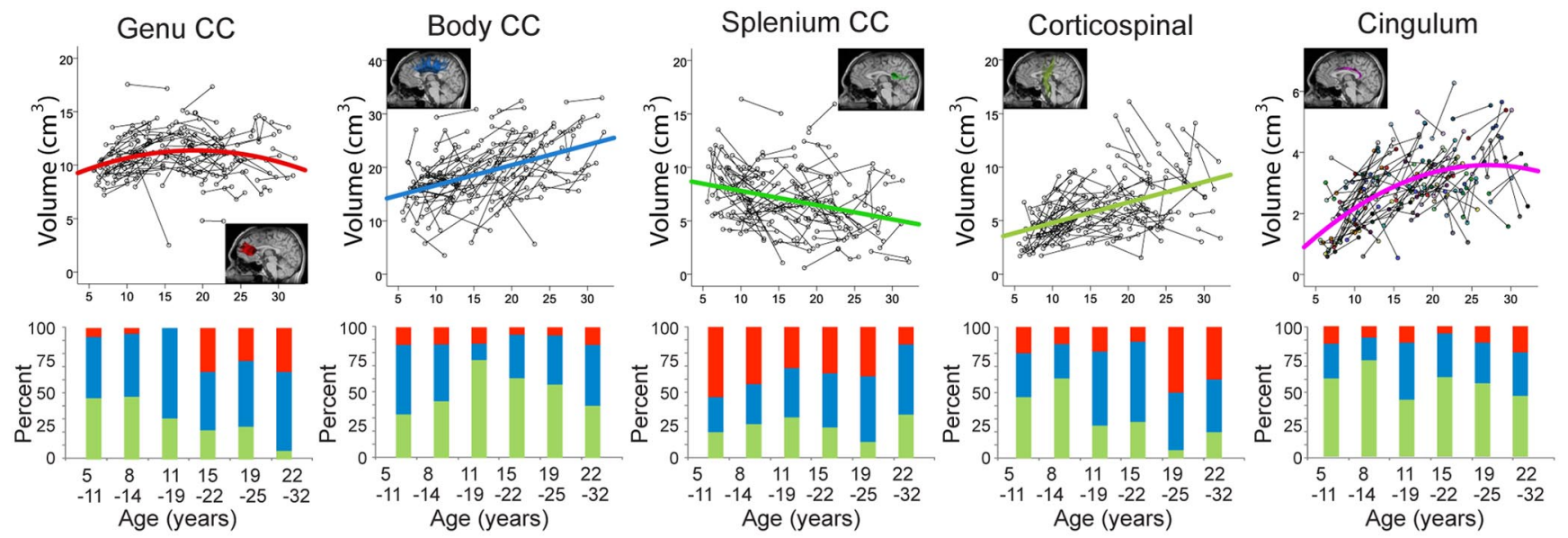

Sup. Longitudinal

Sup. Fronto-occipital

Inf. Fronto-occipital
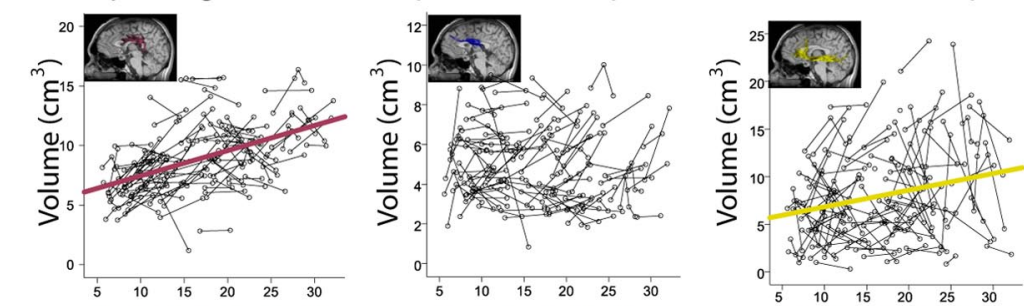

Inf. Longitudinal

Uncinate
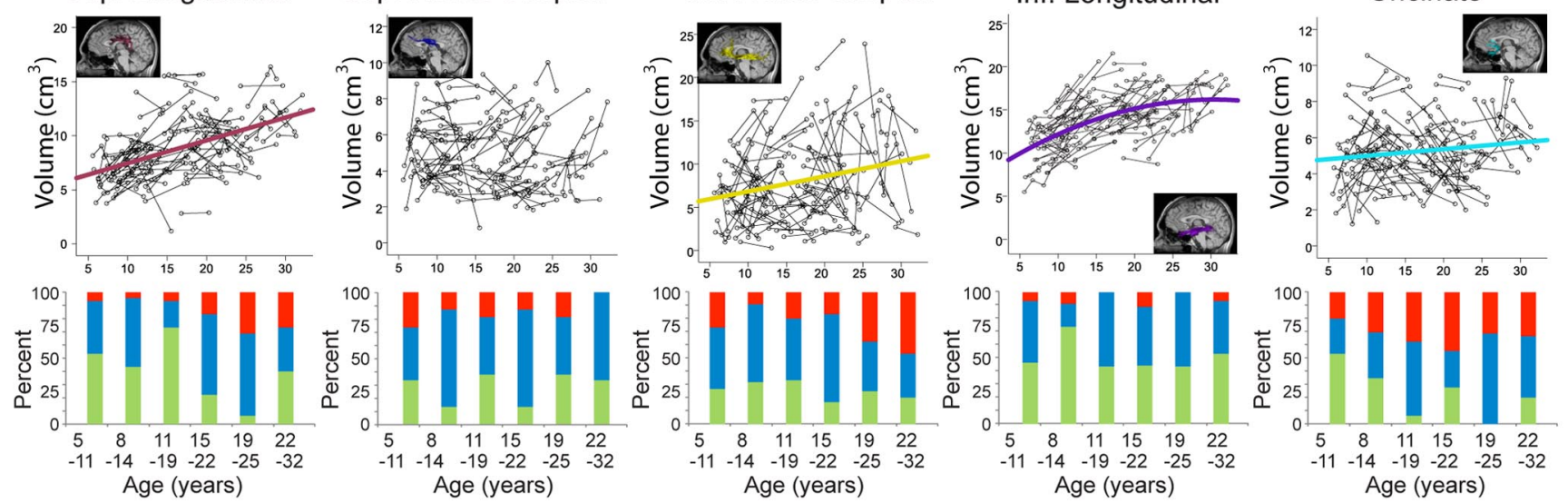

Percent of subjects with: $\quad$ Volume Increase $\quad$ No Change $\quad$ Volume Decrease

Figure 7. Longitudinal changes of tract volume. Volume changes were significant for most tracts, although not for the superior fronto-occipital fasciculus (no trend line shown). Most tracts had linear volume increases across the age range, although the cingulum, genu of the corpus callosum, and the inferior longitudinal fasciculus had quadratic trends with increases then decreases, and the splenium of the corpus callosum showed linear decreases of volume. Note that within-subject increases of tract volumes were observed in $40-50 \%$ of subjects in the $19-25$ and $22-32$ year age groups for the body of the corpus callosum, cingulum, and inferior longitudinal fasciculus.

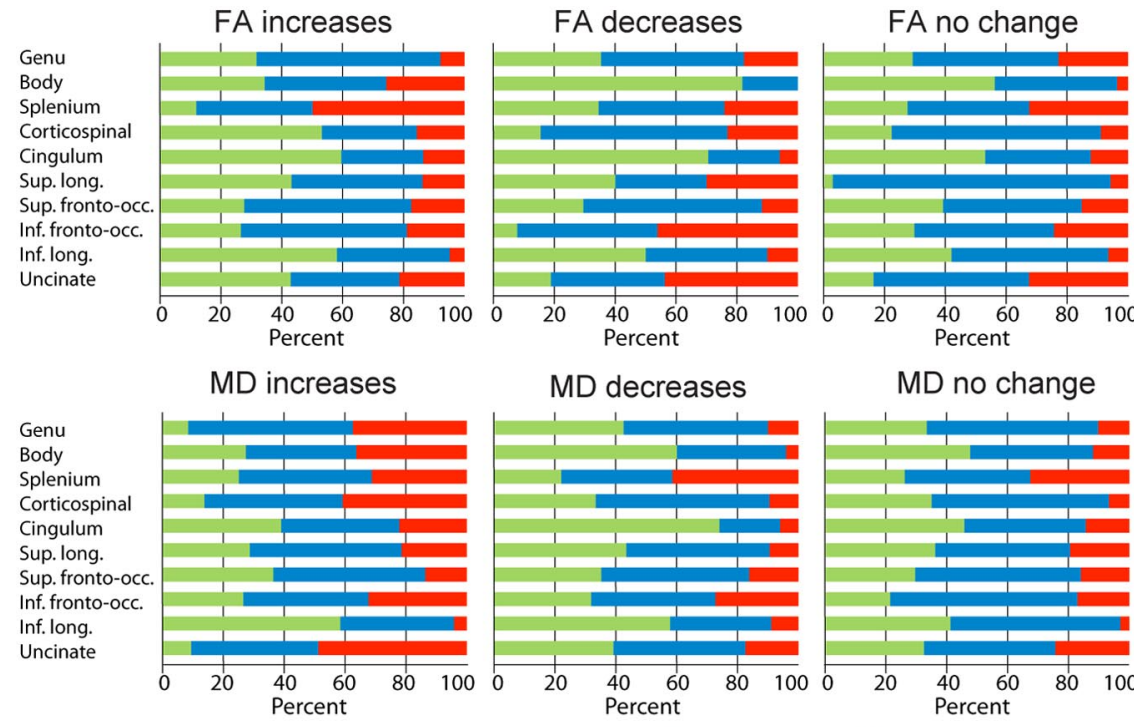

Percent of subjects with: $\quad$ Volume Increase $\quad$ No Change $\quad$ Volume Decrease

Figure 8. Tract volume relationship with FA and MD changes. The percentage of subjects that showed increases (green), decreases (red), or no change (blue) in tract volume between scans are shown for each group depending on the evolution of FA or MD. While there is a decent proportion of subjects with elevated FA or reduced MD that have increased tract volume, there is a greater proportion of subjects with no change or even a decrease in tract volume in thesecases. There is no clear association between tract volume and diffusion parameter changes. ders and underlying brain structure. Many psychiatric disorders emerge during adolescence and young adulthood (Paus et al., 2008), and are related to frontal white matter abnormalities, including the cingulum, uncinate, and superior longitudinal fasciculi in schizophrenia (Kubicki et al., 2007) and the cingulum, inferior and superior longitudinal and fronto-occipital fasciculi in bipolar disorder (Heng et al., 2010). The diversity of postadolescent changes in these association pathways could lead to important studies of risk factors and the role of brain development in various disorders. For example, the uncinate fasciculus undergoes substantial change after adolescence, where $40 \%$ of subjects in the two oldest groups have decreasing FA and 60\% have increasing $\mathrm{MD}$, indicative of interesting microstructural changes that may be relevant to mental health.

Decreasing perpendicular diffusivity between scans drove FA increases in all tracts; parallel diffusivity contributed 
to changes in the corticospinal tract in younger subjects and in some association tracts in adolescents and young adults. While most cross-sectional development studies attribute FA increases to decreasing $\lambda_{\perp}$ (Bonekamp et al., 2007; Eluvathingal et al., 2007; Giorgio et al., 2008; Lebel et al., 2008a; Faria et al., 2010), one ascribes elevated FA to increased $\lambda_{/ /}$from 10 to 20 years (Ashtari et al., 2007). Furthermore, one of the previous two longitudinal DTI studies found that $\lambda_{/ /}$contributed significantly to FA increases during late adolescence (Giorgio et al., 2010). Animal studies show that $\lambda_{\perp}$ is related to myelination and axonal packing (Beaulieu, 2002; Song et al., 2005), whereas $\lambda_{/ /}$can vary with fiber diameter and axon coherence (Takahashi et al., 2000). Although biophysical processes associated with normal human development are more complex than in these animal models (Schmierer et al., 2007; Concha et al., 2010), the decreased $\lambda_{\perp}$ observed here suggests increased myelination and/or more densely packed axons with age. Increased $\lambda_{/ /}$may indicate improved fiber coherence at older ages, as axons straighten and coalesce further within the bundle. Myelination is promoted by electrical impulses across axons, suggesting that training and experience during development may influence myelination and thus inducing the observed diffusion parameter changes (Demerens et al., 1996; Ishibashi et al., 2006).

This study demonstrates longitudinal changes of tract volume, as measured by tractography, for the first time, showing substantial age-related increases in most tracts across the age span (Fig. 7). The body of the corpus callosum, cingulum, and inferior longitudinal fasciculus demonstrated prolonged increases, confirming postadolescent maturation. Tract volume and FA are inherently linked because of the FA tracking threshold, and partial volume effects in typically low-resolution DTI are an additional concern because effects are exaggerated for smaller tracts (Vos et al., 2011). Yet, for all tracts, age remained a significant predictor of FA and MD even after including tract volume as a covariate in mixed-models analysis. Furthermore, similar proportions of individuals with increasing volume were demonstrated among subjects with FA increases and subjects with FA decreases (Fig. 8). Although it is impossible to truly separate tract volume and diffusion parameters, these data suggest that volume is not a direct cause or result of the observed FA and MD changes. Rather, it appears that FA and MD changes are at least partly related to changes of white matter microstructure, such as myelination and increased axonal density. It is important to note that tract volume as measured by DTI does not represent the true volume of the tract, since the edges of tractography bundles are defined artificially by FA values. The representation of tract volume used here is instead a measure of the volume of white matter corresponding to a distinct fiber bundle that exceeds the given FA threshold, in this case, 0.25 .

Observed sex differences were $1-3 \%$, which is small compared with the reliability analysis (SDs of $1.4-3.2 \%$ ) or between-scan changes within individuals (5-7\%). Other longitudinal DTI studies have not detected gender differences (Bava et al., 2010; Giorgio et al., 2010), although volumetric longitudinal studies suggest boys and girls have markedly different development during adolescence (Giedd et al., 1999; Lenroot et al., 2007).

Several limitations must be noted. The quadratic model used to characterize development trajectories may overestimate changes at upper ages since it does not allow for a plateau, but was used because it is simple, easily modeled while accounting for correlated terms, and provides a reasonable approximation to development over most of this age range. Future studies using more sophisticated models may be able to more accurately represent longitudinal diffusion changes within the human brain. Deterministic tractography can be prone to error in low FA regions, such as where fibers cross. This concern is mitigated, however, by the analysis of large, well defined white matter pathways. By necessity, the FA threshold for tracking influences the resulting fiber tracts and their diffusion values. For this study, we used a threshold of 0.25 , which is low enough to allow adequate fiber tracking through white matter, but sufficiently high to prevent tracking through gray matter regions $(\mathrm{FA} \sim 0.2$, although some subcortical gray matter structures have higher FA) and to aid in reducing the presence of spurious fibers.

In this large, longitudinal DTI tractography study, prolonged maturation of white matter fibers beyond adolescence is shown within individual subjects for the first time. The findings of crosssectional studies are extended by showing longitudinally that neural pathway microstructure evolves differentially throughout the white matter in children and adolescents over a 1-6 year span. These diffusion parameter changes are driven primarily by perpendicular diffusivity, suggesting increasing myelination and/or axonal packing not only through the childhood and adolescent periods but also during young adulthood. Normative studies such as this provide valuable information about healthy development, which will be useful for future studies of abnormal development and its relationship to psychiatric disorders that emerge during adolescence and young adulthood.

\section{References}

Ashtari M, Cervellione KL, Hasan KM, Wu J, McIlree C, Kester H, Ardekani BA, Roofeh D, Szeszko PR, Kumra S (2007) White matter development during late adolescence in healthy males: a cross-sectional diffusion tensor imaging study. Neuroimage 35:501-510.

Barnea-Goraly N, Menon V, Eckert M, Tamm L, Bammer R, Karchemskiy A, Dant CC, Reiss AL (2005) White matter development during childhood and adolescence: a cross-sectional diffusion tensor imaging study. Cereb Cortex 15:1848-1854.

Bava S, Thayer R, Jacobus J, Ward M, Jernigan TL, Tapert SF (2010) Longitudinal characterization of white matter maturation during adolescence. Brain Res 1327:38-46.

Beaulieu C (2002) The basis of anisotropic water diffusion in the nervous system - a technical review. NMR Biomed 15:435-455

Ben Bashat D, Ben Sira L, Graif M, Pianka P, Hendler T, Cohen Y, Assaf Y (2005) Normal white matter development from infancy to adulthood: comparing diffusion tensor and high b value diffusion weighted MR images. J Magn Reson Imaging 21:503-511.

Blakemore SJ, Choudhury S (2006) Development of the adolescent brain: implications for executive function and social cognition. J Child Psychol Psychiatry 47:296-312.

Bonekamp D, Nagae LM, Degaonkar M, Matson M, Abdalla WM, Barker PB, Mori S, Horská A (2007) Diffusion tensor imaging in children and adolescents: reproducibility, hemispheric, and age-related differences. Neuroimage 34:733-742.

Concha L, Livy DJ, Beaulieu C, Wheatley BM, Gross DW (2010) In vivo diffusion tensor imaging and histopathology of the fimbria-fornix in temporal lobe epilepsy. J Neurosci 30:996-1002.

Demerens C, Stankoff B, Logak M, Anglade P, Allinquant B, Couraud F, Zalc B, Lubetzki C (1996) Induction of myelination in the central nervous system by electrical activity. Proc Natl Acad Sci U S A 93:9887-9892.

Dubois J, Dehaene-Lambertz G, Perrin M, Mangin JF, Cointepas Y, Duchesnay E, Le Bihan D, Hertz-Pannier L (2008) Asynchrony of the early maturation of white matter bundles in healthy infants: quantitative landmarks revealed noninvasively by diffusion tensor imaging. Hum Brain Mapp 29:14-27.

Eluvathingal TJ, Hasan KM, Kramer L, Fletcher JM, Ewing-Cobbs L (2007) Quantitative diffusion tensor tractography of association and projection fibers in normally developing children and adolescents. Cereb Cortex 17:2760-2768.

Faria AV, Zhang J, Oishi K, Li X, Jiang H, Akhter K, Hermoye L, Lee SK, Hoon 
A, Stashinko E, Miller MI, van Zijl PC, Mori S (2010) Atlas-based analysis of neurodevelopment from infancy to adulthood using diffusion tensor imaging and applications for automated abnormality detection. Neuroimage 52:415-428.

Giedd JN, Blumenthal J, Jeffries NO, Castellanos FX, Liu H, Zijdenbos A, Paus T, Evans AC, Rapoport JL (1999) Brain development during childhood and adolescence: a longitudinal MRI study. Nat Neurosci 2:861-863.

Giorgio A, Watkins KE, Douaud G, James AC, James S, De Stefano N, Matthews PM, Smith SM, Johansen-Berg H (2008) Changes in white matter microstructure during adolescence. Neuroimage 39:52-61.

Giorgio A, Watkins KE, Chadwick M, James S, Winmill L, Douaud G, De Stefano N, Matthews PM, Smith SM, Johansen-Berg H, James AC (2010) Longitudinal changes in grey and white matter during adolescence. Neuroimage 49:94-103.

Heng S, Song AW, Sim K (2010) White matter abnormalities in bipolar disorder: insights from diffusion tensor imaging studies. J Neural Transm 117:639-654.

Hermoye L, Saint-Martin C, Cosnard G, Lee SK, Kim J, Nassogne MC, Menten R, Clapuyt P, Donohue PK, Hua K, Wakana S, Jiang H, van Zijl PC, Mori S (2006) Pediatric diffusion tensor imaging: normal database and observation of the white matter maturation in early childhood. Neuroimage 29:493-504.

Huttenlocher PR (1979) Synaptic density in human frontal cortex- developmental changes and effects of aging. Brain Res 163:195-205.

Ishibashi T, Dakin KA, Stevens B, Lee PR, Kozlov SV, Stewart CL, Fields RD (2006) Astrocytes promote myelination in response to electrical impulses. Neuron 49:823-832.

Jung RE, Haier RJ (2007) The Parieto-Frontal Integration Theory (P-FIT) of intelligence: converging neuroimaging evidence. Behav Brain Sci 30: 135-154; discussion 154-187.

Kim MJ, Whalen PJ (2009) The structural integrity of an amygdalaprefrontal pathway predicts trait anxiety. J Neurosci 29:11614-11618.

Kubicki M, McCarley R, Westin CF, Park HJ, Maier S, Kikinis R, Jolesz FA, Shenton ME (2007) A review of diffusion tensor imaging studies in schizophrenia. J Psychiatr Res 41:15-30.

Lebel C, Walker L, Leemans A, Phillips L, Beaulieu C (2008a) Microstructural maturation of the human brain from childhood to adulthood. Neuroimage 40:1044-1055.

Lebel C, Rasmussen C, Wyper K, Walker L, Andrew G, Yager J, Beaulieu C (2008b) Brain diffusion abnormalities in children with fetal alcohol spectrum disorder. Alcohol Clin Exp Res 32:1732-1740.

Lenroot RK, Gogtay N, Greenstein DK, Wells EM, Wallace GL, Clasen LS, Blumenthal JD, Lerch J, Zijdenbos AP, Evans AC, Thompson PM, Giedd JN (2007) Sexual dimorphism of brain developmental trajectories during childhood and adolescence. Neuroimage 36:1065-1073.

Moll J, Zahn R, de Oliveira-Souza R, Krueger F, Grafman J (2005) Opinion: the neural basis of human moral cognition. Nat Rev Neurosci 6:799-809.

Mukherjee P, Miller JH, Shimony JS, Conturo TE, Lee BC, Almli CR,
McKinstry RC (2001) Normal brain maturation during childhood: developmental trends characterized with diffusion-tensor MR imaging. Radiology 221:349-358.

Paus T, Zijdenbos A, Worsley K, Collins DL, Blumenthal J, Giedd JN, Rapoport JL, Evans AC (1999) Structural maturation of neural pathways in children and adolescents: in vivo study. Science 283:1908-1911.

Paus T, Keshavan M, Giedd JN (2008) Why do many psychiatric disorders emerge during adolescence? Nat Rev Neurosci 9:947-957.

Sala S, Agosta F, Pagani E, Copetti M, Comi G, Filippi M (2010) Microstructural changes and atrophy in brain white matter tracts with aging. Neurobiol Aging. Advance online publication. Retrieved June 29, 2011. doi:10.1016/j.neurobiolaging.2010.04.027.

Schmierer K, Wheeler-Kingshott CA, Boulby PA, Scaravilli F, Altmann DR, Barker GJ, Tofts PS, Miller DH (2007) Diffusion tensor imaging of post mortem multiple sclerosis brain. Neuroimage 35:467-477.

Schmithorst VJ, Wilke M, Dardzinski BJ, Holland SK (2002) Correlation of white matter diffusivity and anisotropy with age during childhood and adolescence: a cross-sectional diffusion-tensor MR imaging study. Radiology 222:212-218.

Scholz J, Klein MC, Behrens TE, Johansen-Berg H (2009) Training induces changes in white-matter architecture. Nat Neurosci 12:1370-1371.

Song SK, Yoshino J, Le TQ, Lin SJ, Sun SW, Cross AH, Armstrong RC (2005) Demyelination increases radial diffusivity in corpus callosum of mouse brain. Neuroimage 26:132-140.

Sowell ER, Thompson PM, Holmes CJ, Jernigan TL, Toga AW (1999) In vivo evidence for post-adolescent brain maturation in frontal and striatal regions. Nat Neurosci 2:859-861.

Sowell ER, Thompson PM, Leonard CM, Welcome SE, Kan E, Toga AW (2004) Longitudinal mapping of cortical thickness and brain growth in normal children. J Neurosci 24:8223-8231.

Takahashi M, Ono J, Harada K, Maeda M, Hackney DB (2000) Diffusional anisotropy in cranial nerves with maturation: quantitative evaluation with diffusion MR imaging in rats. Radiology 216:881-885.

Thompson PM, Giedd JN, Woods RP, MacDonald D, Evans AC, Toga AW (2000) Growth patterns in the developing brain detected by using continuum mechanical tensor maps. Nature 404:190-193.

Vos SB, Jones DK, Viergever MA, Leemans A (2011) Partial volume effect as a hidden covariate in DTI analyses. Neuroimage 55:1566-1576.

Wakana S, Jiang H, Nagae-Poetscher LM, van Zijl PC, Mori S (2004) Fiber tract-based atlas of human white matter anatomy. Radiology 230:77-87.

Westlye LT, Walhovd KB, Dale AM, Bjørnerud A, Due-Tønnessen P, Engvig A, Grydeland H, Tamnes CK, Ostby Y, Fjell AM (2010) Life-span changes of the human brain white matter: diffusion tensor imaging (DTI) and volumetry. Cereb Cortex 20:2055-2068.

Yakovlev PI, Lecours A-R (1967) The myelogenetic cycles of regional maturation of the brain. In: Regional development of the brain early in life (Minkowski A, ed), pp 3-70. Boston: Blackwell Scientific Publications. 